American Journal of Applied Sciences 2 (13): 27-38, 2005

ISSN 1546-9239

(C) 2005 Science Publications

\title{
The Possibility of Cyclical Behavior in a Class of Dynamic Models
}

\author{
Anjan Mukherji \\ Centre for Economic Studies and Planning, Jawaharlal Nehru University, New Delhi
}

\begin{abstract}
The study investigates conditions under which endogenous cyclic behavior may be observed within the context of Predator-Prey (Lotka-Volterra) Models. The analysis also establishes conditions under which such behavior is non-existent and hence establishes conditions for global convergence to the interior equilibrium, whenever it exists. The results are then applied to two diverse sets of economic exercises and shows how the conclusions of those exercises may be established under a much weaker set of assumptions. Based on these discussions, a numerical example of robust periodic behavior is provided.
\end{abstract}

Key words: Predator-prey models, Lotka-Volterra models, robust periodic behavior, Dulac's criterion, Poincaré-Bendixson theorem, Goodwin growth model, Easter Island mystery

\section{INTRODUCTION}

Economic fluctuations are matters of great intrinsic interest and there is a rich history of attempts to provide an explanation for such behavior of particular interest are fluctuations which persist. There have been, basically, two types of explanation: the first relies on exogenous shocks, either from the demand side or from the supply side (See, for instance the contribution of Kydland and Prescott ${ }^{[1]}$, mentioned in the citation of the Nobel Memorial Prize in Economic Sciences in 2004) to explain fluctuations in economic activity; the second approach is to consider whether cyclical behavior could arise from internal or endogenous sources (Examples of this type may be found in the works of Schumpeter ${ }^{[2]}$, Wicksell $^{[3]}$ for example. For Keynesian type models where cyclical behavior stems from an interaction between a multiplier from the consumption side and an accelerator from the investment side, among the more influential have been the contributions by Goodwin ${ }^{[4,5]}$ ). The present paper belongs to the latter category.

Further, we should point out that by cyclical behavior we shall mean persistent cyclical behavior; behavior of trajectories which spiral in towards an equilibrium or steady state is not really proper cyclical behavior since the extent of these fluctuations will eventually die out. Finally, we shall be concerned with models of the Predator-Prey (alternatively, LotkaVolterra) type. These models have been studied by economists since the time Samuelson ${ }^{[6,7]}$ turned his attention on them; one of the most well known applications of this model is the paper by Goodwin ${ }^{[4]}$. Such models continued to be studied and applied (More recent contributions include Mukherji ${ }^{[8]}$ which examines the robustness of cyclical paths in Goodwin ${ }^{[4]}$,
Brander and Taylor ${ }^{[9]}$, who apply the model to analyze the Easter Island Mystery, Cressman et al. ${ }^{[10]}$ who apply this model to study the evolutionary dynamics of crime. There have been applications in areas other than economics $^{[11]}$, as well.

The Predator-Prey Model may be described thus. Consider an environment made up of two species of life-forms, one of which preys on the other: the predator and the prey. Let the population of the prey be designated by $\mathrm{x}$ and that of the predator by $\mathrm{y}$. The simplest formulation in Samuelson ${ }^{[6]}$ or Goodwin ${ }^{[4]}$, for instance, involves the following basic assumption: in the absence of the predator, the population of the prey grows at a constant proportional rate a; and on the other hand, in the absence of the prey, the population of the predator decays at a constant proportional rate $b$ (here both $\mathrm{a}$ and $\mathrm{b}$ are assumed positive). In the presence of both the prey and predator, adjustments to this basic story have to be made and we have the following Eq. (1):

$$
\dot{x}=x(a-\alpha y) \text { and } \dot{y}=y(\beta x-b)
$$

where, $\alpha, \beta$ are assumed to be positive and are to be interpreted as the effect of the presence of one population on the other. We shall refer to (1), as the basic Equations.

There are two equilibria for the above system of equations:

$$
\begin{gathered}
(\mathrm{x}=0, \mathrm{y}=0)\{\text { Trivial Equilibrium or }(\mathrm{TE})\} \\
(\mathrm{x}=\mathrm{b} / \beta, \mathrm{y}=\mathrm{a} / \alpha)\{\text { Non-Trivial Equilibrium or }(\mathrm{NTE})\}
\end{gathered}
$$

We are interested in what happens to the solution to the system (1), $\varphi_{t}\left(\hat{\mathrm{z}}^{0}\right)$ where $\hat{\mathrm{z}}^{0}=\left(\hat{\mathrm{x}}^{0}, \hat{\mathrm{y}}^{\mathrm{o}}\right)$ beginning from an initial configuration $\hat{z}^{\circ}$. In particular, we shall 
be interested in finding out whether any periodic or cyclical behavior is possible.

The paper begins by setting out first, the wellknown dynamics emanating from the basic equations as a benchmark; this is followed by noting the change in results when there is a perturbation in parameter values.

The sharp differences in results make the next step worthwhile; we set-up a general formulation of the Predator-Prey Model and establish that under standard conditions, periodic behavior is non-existent. This also allows us to deduce what changes need to be introduced into the basic structure to allow for periodic behavior. We study the most general form of the predator-prey model; this was investigated by Kolmogorov ${ }^{[12]}$ way back in; a thorough report on these results is contained in Freedman ${ }^{[13]}$. We shall show that our results are more general and our point of focus is different too.

We use the derived results to discuss two applications of the Predator-Prey Model; we show that our methods reveal that the specific assumptions made in these applications are not really needed for the results each have deduced and, in the light of these exercises, we go on to construct an example of a Predator-Prey Model, where robust periodic behavior is exhibited: this example also serves as an illustration of how the framework of the Predator-Prey Model needs to be altered to admit such behavior. A last section contains, by way of conclusion, some discussion of related literature.

The dynamics of the basic equations: We note first of all, the following local stability properties of the equilibria mentioned above. The details can be obtained from Hirsch and Smale ${ }^{[14]}$.

Claim 1: For the system (1), TE is a saddle point while NTE is a center.

Next, we note that, so far as global stability considerations are concerned, we may make the following:

Claim 2: With any $z^{\circ}=\left(x^{\circ}, y^{\circ}\right)>(0,0)$ as initial point, the solution to the system (1) is a closed orbit around NTE.

The above claim may be seen from the following diagram (An analytical proof is provided in Hirsch and Smale ${ }^{[14]}$.

This result has some times been used to explain why the population of some species constantly keep chasing one another and never settles down to any fixed values. We investigate next what happens when we change the basic story a little bit (An investigation into the robustness of the periodic behavior exhibited by the solution to (1) is carried out in Mukherji ${ }^{[8]}$ ).

A perturbation: Suppose that we say that when left to itself, in the absence of the predator, the population of the prey behaves according to a more complicated but still fairly standard rule, the logistic rule:

$$
\dot{\mathrm{x}}=\mathrm{xa}\left(1-\frac{\mathrm{x}}{\mathrm{K}}\right)
$$

where, $\mathrm{K}$ is the carrying capacity of the preys: i.e., the environment cannot sustain a population which is greater than $\mathrm{K}$; in the basic equations, it was assumed that $\mathrm{K}$ is infinite. Thus the environment places a restriction on the growth of population of preys. Now adjusting for the presence of predators, we have (writing $\gamma=\mathrm{a} / \mathrm{K}>0$ ) and keeping the behavior of the predator population unaltered, we have the following Eq. (2):

$\dot{\mathrm{x}}=\mathrm{x}(\mathrm{a}-\gamma \mathrm{x}-\alpha \mathrm{y})$ and $\dot{\mathrm{y}}=\mathrm{y}(\beta \mathrm{x}-\mathrm{b})$

Notice that by setting $\gamma$ equal to zero we revert back to the earlier system. Due to the presence of the term $\gamma$, it may be noted that:

Claim 2: The system (2) has the following three equilibria:

$$
(x=0, y=0)(T E),(x=a / g, y=0)(M P E)
$$

And:

$$
(x=b / b, y=d)(N T E)
$$

Where:

$$
\delta=\frac{\mathrm{a} \beta-\mathrm{b} \gamma}{\alpha \beta}
$$

We assume $\delta$ to be positive. In other words, we have yet another restriction $\gamma<\mathrm{a} \beta / \mathrm{b}$; since we are interested in showing what happens for small values of the parameter $\gamma$, this restriction is not a problem for our analysis. Finally, in the NTE, although the size of the prey is the same as in the earlier case (when $\gamma=0$ ), the size of the predator population is reduced.

We turn next to the stability properties of these equilibria; first, as before, we consider the local stability of equilibria. Once again, details are to be found in Mukherji ${ }^{[8]}$.

Claim 3: For the system (2), TE is a saddle point; MPE is a saddle point while NTE is locally asymptotically stable.

In addition, we have the following global stability result:

Claim 4: For any solution to (2) with a strictly positive initial point converges to the NTE. 


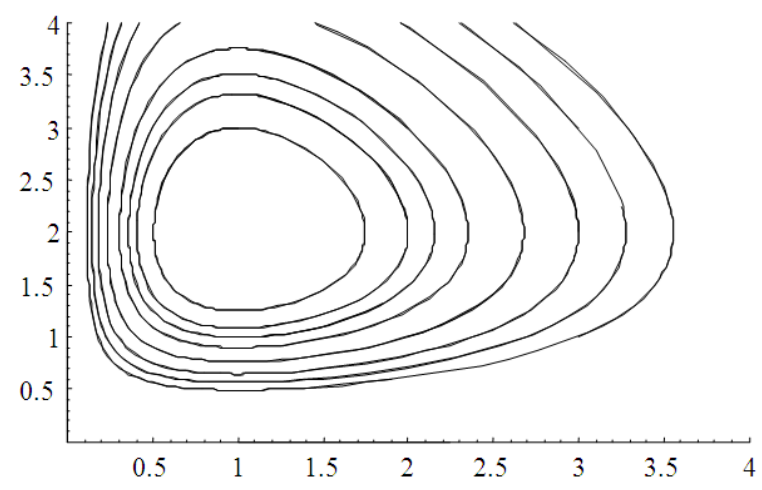

Fig. 1: The predator-prey model $(a=2, b, \alpha, \beta=1)$

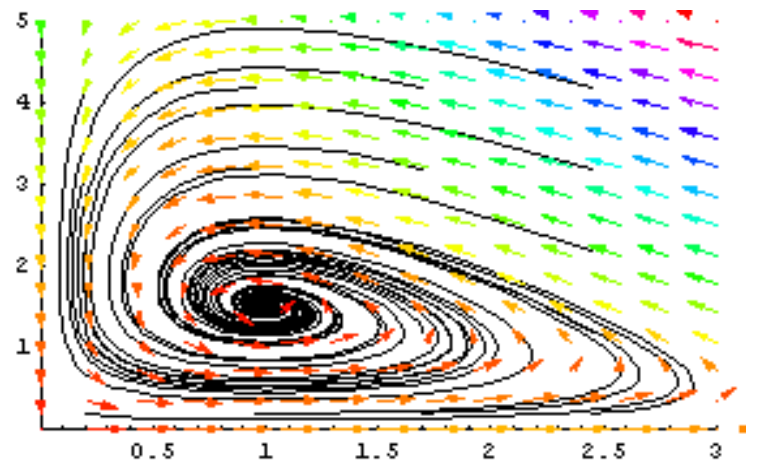

Fig. 2:The predator-prey model (a, b, $\alpha, \beta$ as above; $\gamma$ $=0.5$ )

To clarify the situation further, consider the Figure below. The difference in results due to the presence of the term $\gamma$ is clear when one compares Fig. 2 with the earlier Fig. 1.

To sum up: the dynamic conclusions of the basic equations are very sensitive to the assumption made on the rate of growth of population of preys (or predators). Details of similar exercises may be found in Mukherji ${ }^{[7]}$. In view of this it is meaningful to try to explore whether any general statement can be made about cyclical behavior in such models (the statement in Hirsch and Smale ${ }^{[13]}$, the paragraph just before the statement of the section titled Problem).

A general Lotka-Volterra model: We attempt here to provide a general treatment of Lotka-Volterra Models and identify conditions for periodic behavior and convergence. Apart from being of independent interest, such results might be of specific interest to a very varied set of problems, as we hope to illustrate.

The basic feature of the predator-prey model or the Lotka-Volterra models is a pair of functions. Let $\mathrm{M}, \mathrm{N}$ be two functions $\mathrm{M}, \mathrm{N}: \mathfrak{R}_{+} \times \mathfrak{R}_{+} \rightarrow \mathfrak{R}$ be continuously differentiable $\left(\Re_{+}\right.$denotes the non-negative real line, [0, $\infty]$ ) and satisfy the following:
P1. $\begin{aligned} & \mathrm{M}(0,0)>0,0 \geq N(0,0) ; \mathrm{M}_{\mathrm{y}}(\mathrm{x}, \mathrm{y}) \\ & <0, \mathrm{~N}_{\mathrm{x}}(\mathrm{x}, \mathrm{y})>0 \quad \forall(\mathrm{x}, \mathrm{y}) \in \mathfrak{R}_{+} \times \mathfrak{R}_{+}\end{aligned}$

(A subscript will denote a partial derivative).

P2. $\mathrm{M}_{\mathrm{x}}(\mathrm{x}, \mathrm{y}) \leq 0, \mathrm{~N}_{\mathrm{y}}(\mathrm{x}, \mathrm{y}) \quad \leq 0 \forall(\mathrm{x}, \mathrm{y}) \in \mathfrak{R}_{+} \times \mathfrak{R}_{+}$(Non-zero values of the partials $\mathrm{M}_{\mathrm{x}}, \mathrm{N}_{\mathrm{y}}$ are interpreted as the existence of 'social phenomenon' by Hirsch and Smale ${ }^{[14]}$. P2 ensures that such phenomenon do not increase the rates of growth of population of preys and predators).

A pair of functions M,N satisfying the above two conditions define a Generalized Lotka-Volterra System (GLVS) given by Eq. (3):

$$
\dot{\mathrm{x}}=\mathrm{xM}(\mathrm{x}, \mathrm{y}), \dot{\mathrm{y}}=\mathrm{yN}(\mathrm{x}, \mathrm{y})
$$

As before, the above formulation captures that there are two species in a particular environment, one of which preys on the other (the predator and the prey). The predator requires prey in order to subsist while the prey can live off the environment (this is not taken up for consideration within the model); using the earlier notation, the rates of growth of the population of the species are related as follows: for the prey, the greater the population of the predators, the lower is the prey's rate of growth other things being equal $\mathrm{M}_{\mathrm{y}}<0$; and since the environment too is limited in some way, the rate of growth of the prey, given any fixed level of the population of predators, is decreasing, if at all, in its own population level $\left(M_{x}<0\right)$. For the predator, on the other hand, the rate of growth of its population increases with the population of the prey and decreases with its own population, other things remaining the same. This is the rationale for $\mathbf{P 1}$ and $\mathbf{P 2}$. We call the system (3), a General Lotka-Volterra System. Such a system was first studied by Kolmogorov ${ }^{[12]}$; however as we shall show later, the restrictions employed were stronger (As an example, apparently Kolmogorov required to impose $\mathrm{xN}_{\mathrm{x}}$ $+\mathrm{yN}_{\mathrm{y}}>0$; in Freedman ${ }^{[13]}$ where a Kolmogrov type result is proved, use is made of not only this but many other conditions as well).

We begin by noting that for the system (3) given $\mathbf{P 1}$ and P2, the following are the types of equilibria (A fourth possibility with only predators, is of course impossible under our assumptions):

- No Species $E_{1}:(0,0)$

- No Predator $E_{2}:(\hat{x}, 0), \hat{x}>0$

- Both Species $E_{3}:(\hat{x}, \hat{y})>(0,0)$

Notice first of all, that these equilibria are independent of one another. Consider for example the following specifications of the functions M,N Eq. (4):

$$
M(x, y)=a-\alpha x-b y ; N(x, y)=d x-\beta y-c
$$


where, a, b, c, d >0, $\beta, \alpha \geq 0$. It is immediate to note that.

Claim 6: For the above specification of the functions $M$, $N, E_{2}, E_{3}$ both exist if and only if $\alpha>0 ; \alpha c<\alpha$. If $\alpha=0$, $E_{3}$ exists but $E_{2}$ does not; whereas if $\alpha>0$ but $\alpha \mathrm{c}>$ a d, $\mathrm{E}_{2}$ exists but $\mathrm{E}_{3}$ does not.

Consider, next, the following forms of the functions M,N Eq. (5):

$$
\begin{aligned}
& M(x, y)=\frac{1+a x}{1+x}-\text { by and } N(x, y) \\
& =\frac{1+d x}{1+x}-\beta y-c, 0<a<1, b, \beta>0, d>c>1
\end{aligned}
$$

Note that $\mathrm{M}(0,0)>0>\mathrm{N}(0,0)=1-\mathrm{c} ; \mathrm{M}_{\mathrm{x}}, \mathrm{M}_{\mathrm{y}}<0 ; \mathrm{N}_{\mathrm{x}}>0$, $\mathrm{M}_{\mathrm{x}}, \mathrm{M}_{\mathrm{y}}<0 ; \mathrm{N}_{\mathrm{x}}>0, \mathrm{~N}_{\mathrm{y}}<0$.

Claim 7: For $\mathrm{M}, \mathrm{N}$ given by (5) there is no $\mathrm{E}_{2}$; and $\frac{\mathrm{a}}{\mathrm{b}}>\frac{\mathrm{d}-\mathrm{c}}{\beta} \Rightarrow$ there is no $\mathrm{E}_{3}$.

Proof: The first part of the claim is immediate since $\mathrm{M}(\mathrm{x}, 0)=0$ has no positive solution. Notice that along $M(x, y)=0$, we have $y>a / b$.

Similar considerations along $\mathrm{N}(\mathrm{x}, \mathrm{y})=0$ lead us to conclude that along this curve, $y>(d-c / \beta$. Consequently the claim follows.

The above discussion goes to show that the existence of equilibria $E_{2}$ and $E_{3}$ are independent of one another and we need to strengthen P2 in order to specify existing equilibria. We do so below:

i. $\mathrm{M}_{\mathrm{x}}(\mathrm{x}, \mathrm{y})<0, \mathrm{~N}_{\mathrm{y}}(\mathrm{x}, \mathrm{y})<0 \forall(\mathrm{x}, \mathrm{y}) \in \mathfrak{R}_{+} \times \Re_{+}$;

ii. There is some positive integer $K$ such that

P3. $M(x, y) \leq 0$ if either $x \geq K$ or $y \geq K$;

iii. For any $y \geq 0, \exists x(y) \ni N(x(y), y)>0$;

futher for each $x \geq 0, \exists K(x)$ э $y \geq K(x) \Rightarrow N(x, y) \leq 0$.

To maintain our analogy with $x$ being the population of prey and $y$ being the population of predators, the above specifies that first of all, the rates of growth of population of preys and predators are decreasing functions of their own population, other things remaining the same; $\mathbf{P 2}$ had merely required that these be non-increasing functions. Secondly, if either the population of preys or that of the predators are large enough, the rate of growth of population of preys cannot be positive; and finally, for the growth rate of the population of predators to be positive, given any current level of its population, requires an adequate population of preys; and given any population of preys, if the population of predators is large enough, the growth rate of the predator population will be nonpositive. That these requirements are not too restrictive may be seen by referring to the specification given by
(4) when all the parameters a,b,c,d $\alpha, \beta$ are positive: this system satisfies P1 and P3. We note that:

Claim 8: Under $\mathbf{P 1}$ and P3, there are at most three equilibria; $\mathrm{E}_{1}, \mathrm{E}_{2}$ always exist; $\mathrm{E}_{3}$ may also exist under some conditions (Essentially, the curves $\mathrm{M}(\mathrm{x}, \mathrm{y})=0$, $\mathrm{N}(\mathrm{x}, \mathrm{y})=0$ must intersect in the positive orthant).

Proof: We have already seen that there are three types of equilibria possible; $\mathbf{P 3}$ ensures that there cannot be multiple equilibria of any type, since intersections between the curves $\mathrm{M}(\mathrm{x}, \mathrm{y})=0, \mathrm{~N}(\mathrm{x}, \mathrm{y})=0$, the $\mathrm{x}$-axis, the $y$-axis is unique if at all; since the first has a negative slope while the second has a positive slope:

$$
\left.\frac{\mathrm{dy}}{\mathrm{dx}}\right|_{\mathrm{M}(\mathrm{x}, \mathrm{y})=0}=\frac{-\mathrm{M}_{\mathrm{x}}}{\mathrm{M}_{\mathrm{y}}}<0 ;\left.\frac{\mathrm{dy}}{\mathrm{dx}}\right|_{\mathrm{N}(\mathrm{x}, \mathrm{y})=0}=\frac{-\mathrm{N}_{\mathrm{x}}}{\mathrm{N}_{\mathrm{y}}}>0
$$

Hence there can be at most three equilibria.

Since $\mathrm{M}(0,0)>0 \geq \mathrm{M}(\mathrm{K}, 0)$ there is some $\hat{\mathrm{x}} \in(0, \mathrm{~K}]$ such that $\mathrm{M}(\hat{\mathrm{x}}, 0)=0 \Rightarrow(\hat{\mathrm{x}}, 0)$ is the $\mathrm{E}_{2}$ equilibrium with no predators.

Next by virtue of the restriction placed on the function $N(x, y)$ we note that there is $x(0)$ such that $\mathrm{N}(\mathrm{x}(0), 0)>0 \geq \mathrm{N}(0,0)$ and hence there is $\overline{\mathrm{x}} \in[0, \mathrm{x}(0)) \ni \mathrm{N}(\overline{\mathrm{x}}, 0)=0$. If $\overline{\mathrm{x}}>\hat{\mathrm{x}}$ then an $\mathrm{E}_{3}$ equilibrium exists; to see this, note that under this condition $\mathrm{M}(\overline{\mathrm{x}}, 0)>\mathrm{M}(\hat{\mathrm{x}}, 0)=0=\mathrm{N}(\overline{\mathrm{x}}, 0), \quad$ while $\mathrm{N}(\hat{\mathrm{x}}, 0)>\mathrm{N}(\overline{\mathrm{x}}, 0)=0=\mathrm{M}(\hat{\mathrm{x}}, 0)$; thus from continuity, there has to be some $\tilde{x}>\bar{x}$ and $\hat{x}>\tilde{x}$ and some corresponding $\tilde{y}>0$ such that $M(\tilde{x}, \tilde{y})=N(\tilde{x}, \tilde{y})$ : the $E_{3}$ equilibrium.

Local stability properties of equilibria: We examine the local stability of the equilibria $E_{1}, E_{2}$ and $E_{3}$ in this section. First of all, we need to compute the Jacobian of the system (3) given P1 and P2:

$$
\left(\begin{array}{cc}
\mathrm{M}(\mathrm{x}, \mathrm{y})+\mathrm{xM}_{\mathrm{x}}(\mathrm{x}, \mathrm{y}) & \mathrm{xM}_{\mathrm{y}}(\mathrm{x}, \mathrm{y}) \\
\mathrm{yN}_{\mathrm{x}}(\mathrm{x}, \mathrm{y}) & \mathrm{N}(\mathrm{x}, \mathrm{y})+\mathrm{yN}_{\mathrm{y}}(\mathrm{x}, \mathrm{y})
\end{array}\right)
$$

Thus at $\mathrm{E}_{1}:(0,0)$ the above reduces to:

$$
\left(\begin{array}{cc}
+ & 0 \\
0 & -
\end{array}\right)
$$

This establishes that at $\mathrm{E}_{1}$, the Jacobian has one positive and one negative characteristic root and hence the equilibrium is a saddle-point. Next at $E_{2}$, the Jacobian is given by: 


$$
\left(\begin{array}{cc}
- & - \\
0 & \mathrm{~N}(\hat{\mathrm{x}}, 0)
\end{array}\right)
$$

The sign of the term $\mathrm{N}(\hat{\mathrm{x}}, 0)$ depends on whether $\hat{\mathrm{X}}>\overline{\mathrm{X}}$ : the condition for the existence of $\mathrm{E}_{3}$ as we had shown in the proof of Claim 8 . Since by definition, $\mathrm{N}(\overline{\mathrm{x}}, 0)=0$, if $\mathrm{E}_{3}$ exists, $\mathrm{E}_{2}$ is a saddle point, since then one characteristic root is positive and the other negative; whereas if there is no $E_{3}$ equilibrium, then $E_{2}$ is locally asymptotically stable since both characteristic roots are then negative.

Whenever $E_{3}$ exists, the Jacobian evaluated at $E_{3}$ has the following sign pattern:

$$
\left(\begin{array}{ll}
- & - \\
+ & -
\end{array}\right)
$$

Consequently, whenever $E_{3}$ exists, the Jacobian has both roots with real parts negative and the equilibrium is locally asymptotically stable.

Finally, consider the system (1); we may consider this to be a special case of (4) where $\alpha=\beta=0$. It is easy to check that under this restriction, the system (4) (or the system (1) (Notice that there has been a change in notation from the one used in (1)) has two equilibria. $E_{1}:(0,0)$ and $E_{3}:\left(\frac{c}{d}, \frac{a}{b}\right)$ and at $E_{3}$, the Jacobian has the following sign pattern:

$$
\left(\begin{array}{ll}
0 & - \\
+ & 0
\end{array}\right)
$$

Thus under (1), the characteristic roots are pure complex, the real parts being zero. Hence $\mathrm{E}_{3}$ is a center.

\section{Global stability properties:}

Invariant set: For the system (3) we shall refer to a solution originating from some point $\left(\mathrm{x}^{\mathrm{O}}, \mathrm{y}^{\mathrm{O}}\right)$ by the notation $\varphi_{\mathrm{t}}\left(\mathrm{x}^{\mathrm{O}}, \mathrm{y}^{\mathrm{O}}\right)$ and our objective here is to tie down what happens to this solution as $t \rightarrow \infty$. We show that under our assumptions $\mathbf{P 1}$ and $\mathbf{P 3}$, there is an invariant set $\mathrm{Q} \subseteq \mathfrak{R}_{+} \times \mathfrak{R}^{+}$. That is, the solution $\varphi_{\mathrm{t}}\left(\mathrm{x}^{\mathrm{O}}, \mathrm{y}^{\mathrm{O}}\right)$ is defined for all $(x, y) \in Q$ and remains within the set $Q$ for all $t$. We do this constructively in the following steps.

From P1 and P3, we conclude: $\mathrm{M}(\mathrm{x}, \mathrm{y})=0, \mathrm{~N}(\mathrm{x}, \mathrm{y})$ $=0$ are respectively downward sloping and upward rising curves. Also, note that $\mathrm{M}(0,0)>0 \geq \mathrm{M}(0, \mathrm{~K})$; hence, $\exists \bar{y} \in\left(0, K\right.$ such that $\mathrm{M}(0, \overline{\mathrm{y}})=0$. Note that $\mathrm{E}_{2}$ exists; denote this by $(\hat{\mathrm{x}}, 0)$.

Given $\bar{y}$ noted above, $\mathbf{P 3}$ guarantees that there is $\mathrm{x}(\overline{\mathrm{y}})$ such that $\mathrm{N}(\mathrm{x}(\overline{\mathrm{y}}), \overline{\mathrm{y}})=0$.

Define $x^{*}=\operatorname{Max}[\hat{x}, x(\bar{y})]$; next consider, $y^{*}$ such that $\mathrm{N}\left(\mathrm{x}^{*}, \mathrm{y}^{*}\right)=0$; that this is well defined may be seen as follows.
Note that $x^{*}=x(\bar{y}) \Rightarrow y^{*}=\bar{y}$. On the other hand, $\mathrm{x}^{*}=\hat{\mathrm{x}}>\mathrm{x}(\overline{\mathrm{y}}) \Rightarrow \mathrm{N}(\hat{\mathrm{x}}, \overline{\mathrm{y}})>0$ (Since $\mathrm{N}(.,$.$) is increasing$ in $\mathrm{x}$ and $N(x(\bar{y}), \bar{y})=0)$. Thus by $\mathbf{P 3}$, there is $\mathrm{K}(\hat{\mathrm{x}})$ such that $N(\hat{x}, y) \leq 0 \forall y \geq K(\hat{x})$; hence there is $\mathrm{y}^{*}$ such that $\mathrm{N}\left(\hat{\mathrm{x}}, \mathrm{y}^{*}\right)=0$ as claimed.

Now consider the rectangle $Q$ made up with the points $\mathrm{E}_{1}:(0,0),\left(0, \mathrm{y}^{*}\right),\left(\mathrm{x}^{*}, \mathrm{y}^{*}\right),\left(0, \mathrm{x}^{*}\right)$.

We may now show:

Claim 9: The set $Q$ defined above is invariant.

Proof: Consider any $(x, y) \in Q_{B}$ where $Q_{B} \subseteq Q$ is the boundary of $Q$ and consider the solution (trajectory) $\varphi_{\mathrm{t}}(\mathrm{x}, \mathrm{y})$. We shall show that the trajectory either coincides with the boundary or enters $Q$.

$E_{1}$ is an equilibrium so any trajectory originating there stays put; in case $x^{*}=\hat{x}$, the point $\left(x^{*}, 0\right)$ is another equilibrium, $\mathrm{E}_{2}$ and once again, any trajectory originating there stays put; in case $x^{*}=x(\bar{y})>\hat{x}$, any trajectory originating from $\left(x^{*}, 0\right)$ has $\dot{y}=0, \dot{x}<0$,

Since $\mathrm{M}\left(\mathrm{x}^{*}, 0\right)<\mathrm{M}(\hat{\mathrm{x}}, 0)=0$ and so the trajectory coincides with the $x$-axis, and is directed inside $Q$ along the boundary. Notice that any trajectory originating from a point on the $x$-axis is directed along the axis towards the equilibrium $\mathrm{E}_{2}$ and hence stays within $Q$.

Consider, next, any point $(0, y)$ with $0<y \leq y^{*}$; the trajectory originating from such a point has $\dot{\mathrm{x}}=0, \dot{\mathrm{y}}<0 \operatorname{sinceN}(0, \mathrm{y})<0$ and hence the trajectory coincides with the $y$-axis and is directed towards $\mathrm{E}_{1}$.

Any trajectory originating from a point of the type $\left(\mathrm{x}, \mathrm{y}^{*}\right), \quad 0<\mathrm{x} \leq \mathrm{x} *$ has $\dot{\mathrm{x}}<0, \dot{\mathrm{y}}<0$ since $\mathrm{M}\left(\mathrm{x}, \mathrm{y}^{*}\right)<0$, $\mathrm{N}\left(\mathrm{x}, \mathrm{y}^{*}\right)<0$ and consequently, the trajectory is directed inside Q. Similarly, any trajectory originating from a point of the type $\left(x, y^{*}\right), 0<y \leq y^{*}$ is also directed inside $\mathrm{Q}$. This completes the demonstration of our claim.

Figure $3 \mathrm{~A}$ and $3 \mathrm{~B}$ would clarify the claims made above (Although the lines $\mathrm{M}(\mathrm{x}, \mathrm{y})=0, \mathrm{~N}(\mathrm{x}, \mathrm{y})=0$ have been drawn as straight lines they need not be so; it is their slopes which are of importance).

General conclusion: By appealing to the PoincaréBendixson Theorem, the following conclusions may be noted (The investigations of Kolmogorov ${ }^{[12]}$, as reported in Freedman ${ }^{[13]}$, lead to Remarks $1-3$ but it should be pointed out that the conditions P1 and P2, which are the basic conditions employed in this study are weaker than the conditions employed in Kolmogorov's discussion and the proof provided in Freedman ${ }^{[13]}$ ).

Remark 1: For any $(\mathrm{x}, \mathrm{y}) \in \mathfrak{R}_{+} \times \mathfrak{R}_{+}$the trajectory $\varphi_{\mathrm{t}}(\mathrm{x}, \mathrm{y})$ must enter $\mathrm{Q}$ and either approaches equilibrium or there 
is a limit cycle in Q. Any limit cycle, if there is one, must surround an equilibrium.

Remark 2: Further, when there is no $\mathrm{E}_{3}$, as in Fig. $3 \mathrm{~A}$, the triangular region bounded by the axes and the curve $\mathrm{M}(\mathrm{x}, \mathrm{y})=0$ is an invariant subset of $\mathrm{Q}$ : once entered, it cannot be left. In such a situation, there can be no limit cycle and hence any trajectory must approach the equilibrium $\mathrm{E}_{2}$. Consequently, ultimately, all predators disappear.

Remark 3: On the other hand, when we have an equilibrium such as $\mathrm{E}_{3}$ as in Fig. $3 \mathrm{~B}$, there appear to be only two possibilities for any trajectory: beginning from a strictly positive configuration: either we have convergence to $\mathrm{E}_{3}$ or a limit cycle around $\mathrm{E}_{3}$. Neither $\mathrm{E}_{1}$ nor $\mathrm{E}_{2} \mathrm{can}$ be approached.

However, given $\mathbf{P 3}$, we can go further. Recall that under this restriction, we have first of all that $\mathrm{M}_{\mathrm{x}}<0$, $\mathrm{N}_{\mathrm{y}}<0$ over the domain of discussion. Then consider the function $\theta(x, y)=\frac{1}{x \cdot y}$ in the positive quadrant and consider the expression:

$$
\begin{aligned}
& \frac{\partial(\theta(x, y) \cdot x M(x, y))}{\partial x}+\frac{\partial(\theta(x, y) \cdot y N(x, y))}{\partial y} \\
& =\frac{1}{y} M_{x}(x, y)+\frac{1}{x} N_{y}(x, y)<0
\end{aligned}
$$

Over the entire positive quadrant: Hence by Dulac's criterion (Dulac's Criterion: If there exists a function $\theta(\mathrm{x}, \mathrm{y})$ continuously differentiable on the region $S$ such that the expression: $\frac{\partial(\theta(\mathrm{x}, \mathrm{y}) \cdot \mathrm{xM}(\mathrm{x}, \mathrm{y}))}{\partial \mathrm{x}}+\frac{\partial(\theta(\mathrm{x}, \mathrm{y}) \cdot \mathrm{yN}(\mathrm{x}, \mathrm{y}))}{\partial \mathrm{y}}$ is not identically zero on $\mathrm{S}$, and is of constant sign, then there can be no closed orbits of the system in S. Notice that the requirement is that the above expression is $\leq$ or $\geq 0$ but not 0 everywhere.), there can be no cycles in the positive quadrant.

Thus when there is no equilibrium with 'Both Species' present, all trajectories converge to the 'No Predator' equilibrium; if equilibrium with 'Both Species' present exists, we will have convergence to this equilibrium. To apply Dulac's criterion, it may be recalled that we do not need the strict signs of the partial derivatives $M_{x}, N_{y}$; the weak signs admitted under the restriction P2 are sufficient together with a proviso that they are not identically zero. Consequently, if we are interested in exhibiting cyclical behavior of any kind, we must have some variation in the sign of $\mathrm{M}_{\mathrm{x}}, \mathrm{N}_{\mathrm{y}}$. In particular, we may state (There is thus no possibility of cyclic orbits under the (Kolmogorov ${ }^{[12]}$ or Freedman ${ }^{[13]}$ assumptions):

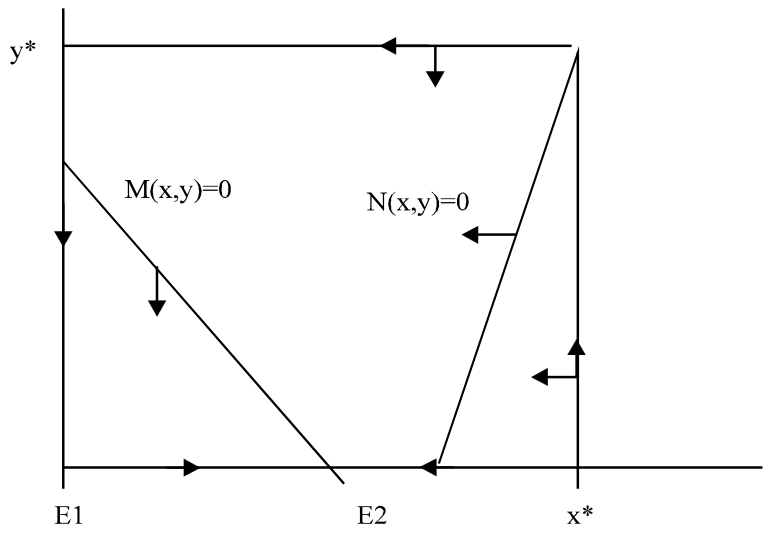

Fig. 3A: The Invariant Set When there is no E3

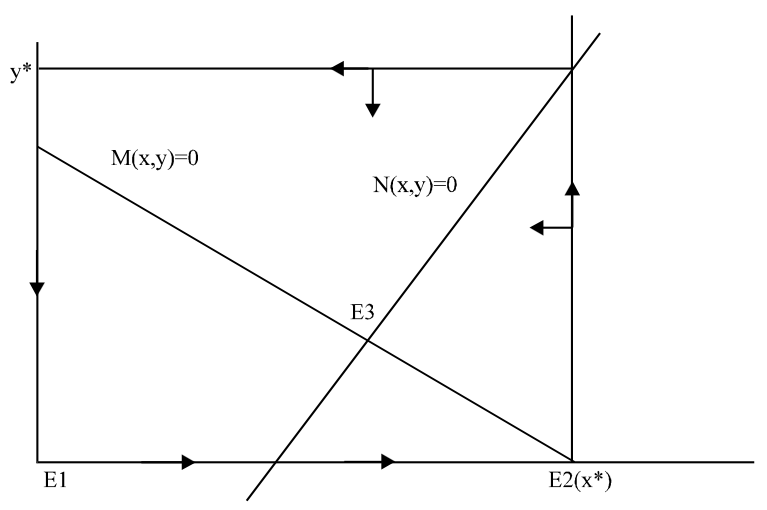

Fig. 3B: The Invariant Set when there is E3

Claim 10: A necessary conditions for the existence of periodic or cyclical trajectories to (3) is that $\mathrm{xM}_{\mathrm{x}}+\mathrm{yN}_{\mathrm{y}}$ change signs on the positive orthant.

It should also be clear from our discussion that the restriction P3 is not really essential in its entirety, for our conclusions to follow: The only use made of $\mathbf{P 3}$ (ii), (iii) was to demonstrate that trajectories remain within some bounded region. We may note this for future reference thus:

Claim 11: Under P1 and P3(i) if the solutions to (3) remain bounded and if either $\mathrm{M}_{\mathrm{x}}$ or $\mathrm{N}_{\mathrm{y}}$ is not identically zero, then there can be no cyclical or periodic trajectory.

A final appeal to the Poincaré-Bendixson Theorem, allows us to note the following set of sufficient conditions for the existence of cyclical behavior:

Claim 12: If any solution to (3) remain bounded, 'Both Species' present Equilibrium $\mathrm{E}_{3}$ exists and $\hat{\mathrm{x}} \mathrm{M}_{\mathrm{X}}(\hat{\mathrm{x}}, \hat{\mathrm{y}})+\hat{\mathrm{y}} \mathrm{N}_{\mathrm{y}}(\hat{\mathrm{x}}, \hat{\mathrm{y}}) \geq 0$, where all terms are evaluated at $E_{3}$, then the solution to (3) would exhibit cyclical behavior i.e., either the solution is a closed orbit or there is a limit cycle. 
We shall use the above claims to provide an example of a Predator-Prey Model with robust cyclical behavior later on. First we show how our method allows us to obtain results which are a lot more general than the existing ones.

\section{Applications}

\section{Stability in a Goodwin-type growth model}

The Goodwin growth model: The Goodwin ${ }^{[4]}$ contribution was perhaps one of the more influential studies on growth theory. The model is based on the following assumptions:

- Steady disembodied technical progress

- Steady growth in labor force

- Only two factors of production, labor and 'capital'

- All quantities are real and net

- All wages are consumed and all profits are saved

- A constant capital output ratio

- A real wage rate which rises in the neighborhood of full employment

The following notation is used: ' $q$ ' denotes output; ' $\mathrm{k}$ ' is capital; ' $w$ ' is the wage rate; $\alpha=\alpha_{O} \mathrm{e}^{\alpha \mathrm{t}}$ is labor productivity growth, $\alpha$ is a constant as specified by (i); the constant capital-output ratio is $\sigma$; $\mathrm{u}$, the share of workers in total product $=$ w/a; naturally the share of the capitalists' is 1-w/a; investment is given by $\dot{\mathrm{k}}=(1-\mathrm{w} / \mathrm{a}) \mathrm{q} ; \ell$, employment is then $\mathrm{q} / \mathrm{a}$; labor $\mathrm{n}$ at time $\mathrm{t}$ is given by $\mathrm{n}_{\mathrm{O}} \mathrm{e}^{\beta \mathrm{t}}$ where $\beta$ is constant. The employment ratio is given by $v=\ell / n$. Finally (vii) is captured by the equation Eq. (6):

$$
\frac{\dot{\mathrm{w}}}{\mathrm{w}}=\mathrm{f}(\mathrm{v})=-\gamma+\rho \mathrm{v}
$$

Goodwin pointed out, in this set-up, that, first of all, $\dot{\ell} / \ell=(1-w / a) / \sigma-\alpha$ so that Eq. (7):

$$
\frac{\dot{\mathrm{v}}}{\mathrm{v}}=\frac{1-\mathrm{u}}{\sigma}-(\alpha+\beta)
$$

And further using the assumption contained in (6), we have Eq. (8):

$$
\frac{\dot{\mathrm{u}}}{\mathrm{u}}=-(\gamma+\alpha)+\rho \mathrm{v}
$$

It should be clear that the system of equations made up of (7) and (8) may be written as Eq. (9):

$$
\left(\begin{array}{c}
\dot{v} \\
\dot{u}
\end{array}\right)=\left(\begin{array}{c}
\mathrm{v}\left\{\frac{1-\mathrm{u}}{\sigma}-(\alpha+\beta)\right\} \\
\mathrm{u}\{-(\gamma+\alpha)+\rho \mathrm{v}\}
\end{array}\right)
$$

These equations constitute a Lotka Volterra system of the type we analyzed above. Consequently, for any arbitrary initial point $\left(v^{\mathrm{O}}, \mathrm{u}^{\mathrm{O}}\right)>(0,0)$, the above system of equations generates a closed orbit around the NTE. The situation depicted in Fig. 1 applies and so does the analysis.

A modification: Before passing on to other matters, it should be pointed out that possibilities of convergence in Goodwin type models have been noted (Mukherji ${ }^{[8]}$ contains a detailed analysis of perturbation exercises within this model). An exercise due to Flaschel ${ }^{[15]}$ may be reported to indicate the benefits obtained from the approach adopted in the present paper.

Flaschel considers the following variation to the Goodwin basic model:

$$
\frac{\dot{\mathrm{w}}}{\mathrm{w}}+\eta \pi=\mathrm{f}(\mathrm{v}), \mathrm{f}^{\prime}(\mathrm{v})>0
$$

where, $\pi=g(u), g^{\prime}(u)>0, g(0)=0$ to denote that the rate of inflation $\pi$ is estimated, as a constant mark-up over labor unit-costs $u$; in addition, assuming a constant output-capital ratio as before, we have:

$$
\frac{\dot{\mathrm{K}}}{\mathrm{K}}=\mathrm{s}(\mathrm{u}) \mathrm{Y} / \mathrm{K}=\mathrm{s}(\mathrm{u}) / \sigma, \mathrm{s}^{\prime}(\mathrm{u})<0
$$

Thus, in contrast to what we have described as the original Goodwin formulation, the Phillips curve (6) has been adjusted for "money illusion" so that when $\eta>0(<0)$ workers receive a lower (respectively, higher) real wage than they bargained for; and the investment or accumulation equation is a straightforward generalization from the constant savings rate assumption. Combining these, we have the following system Eq. (10):

$$
\left(\begin{array}{c}
\dot{\mathrm{v}} \\
\dot{\mathrm{u}}
\end{array}\right)=\left(\begin{array}{c}
\mathrm{v}\{\mathrm{s}(\mathrm{u}) / \sigma-(\alpha+\beta)\} \\
\mathrm{u}\{\mathrm{f}(\mathrm{v})-\alpha-\eta \mathrm{g}(\mathrm{u})\}
\end{array}\right)
$$

The difference between (9) and the above is easy to spot: in fact if we were to replace the functions $\mathrm{g}(),. \mathrm{s}($.$) by$ linear affine approximations, then (10) could be seen as a perturbation of the system (9) for non-zero values of $\eta$; in this sense, the relationship is similar to the one between (1) and (2) except that in the present case, it is the rate of growth of the 'predators' which have been perturbed; hence we expect convergence once again (In this sense, 
the Flaschel ${ }^{[15]}$ enquiry is similar to the one carried out in Mukherji ${ }^{[8]}$; however, the results obtained are different).

For the system (10), the following result is claimed by Flaschel ${ }^{[15]}$ :

Assume that the Jacobian $\mathrm{J}=\left(\mathrm{J}_{\mathrm{ik}}\right)$ of the system (10) satisfies the following:

- $\quad$ Trace $\mathrm{J}<0$,

- $\quad$ Det $\mathrm{J}>0$ and

- $\mathrm{J}_{12} \cdot \mathrm{J}_{21} \neq 0$

Everywhere on $\mathfrak{R}_{+}^{2}$, then the equilibrium $\mathrm{u}^{*}, \mathrm{v}^{*}$ is asymptotically stable in the large.

Now $\mathbf{J}$ is given by the following matrix:

$$
\left(\begin{array}{cc}
\mathrm{s}(\mathrm{u}) / \sigma-(\beta+\alpha) & \mathrm{vs}^{\prime}(\mathrm{u}) / \sigma \\
\mathrm{uf}^{\prime}(\mathrm{v}) & \mathrm{f}(\mathrm{v})-(\alpha+\eta)
\end{array}\right)
$$

It should be easy to see that at the equilibrium, all the conditions mentioned above are satisfied; notice too that the only condition which is easily seen to hold all over the non-negative orthant is the requirement on the off-diagonal terms, given the sign restrictions on the derivatives of the functions $\mathrm{f}(),. \mathrm{s}($.$) ; it is not at all clear how the other two$ requirements on the trace and the determinant are going to be met on the entire non-negative orthant and hence these are demanding restrictions.

However without imposing any of the above requirements (Notice too that the conclusions do not depend upon the form of the functions $\mathrm{f}(\mathrm{v}), \mathrm{s}(\mathrm{u}), \mathrm{g}(\mathrm{u})$; the crucial aspect is the fact that g'(u) is of constant sign. Flaschel ${ }^{[15]}$ proceeds some what differently by transforming variables $\mathrm{x}=\operatorname{lnu}, \mathrm{y}=\operatorname{lnv}$; on $\mathrm{p}$. 65 , middle of the page, the Jacobian for the transformed equations are considered and it is noted that ".. it fulfills the same conditions as were postulated with regard to J." The 'postulated' conditions are redundant even there since the transformed Jacobian has the sign pattern $\left(\begin{array}{l}-+ \\ -\end{array}\right)$ which satisfies all the requirements of olech's theorem.), notice that one may claim:

Claim 13: For the system (10), there can be no cyclical orbits in the positive orthant, given the sign restrictions on the derivatives of the functions $g$.

Proof: Consider the function $\theta(v, u)=1 / v \cdot u$ and then consider:

$$
\begin{aligned}
& \frac{\partial\{\theta(\mathrm{v}, \mathrm{u}) \cdot \mathrm{v}(\mathrm{s}(\mathrm{u}) / \sigma-(\alpha+\beta))\}}{\partial \mathrm{v}} \\
& +\frac{\partial\{\theta(\mathrm{v}, \mathrm{u}) \mathrm{u}(\mathrm{f}(\mathrm{v})-\alpha-\eta \mathrm{g}(\mathrm{u}))\}}{\partial \mathrm{u}}=-\eta \mathrm{g}^{\prime}(\mathrm{u})
\end{aligned}
$$

This has the same sign (non-zero) on the positive quadrant: hence by Dulac's Criterion, there can be no cycles in the positive orthant.

Thus for convergence to equilibrium from any initial positive configuration, one may note that additionally we need to show that the solution is bounded and one may use the Poincaré-Bendixson Theorem to complete the demonstration. It may be recalled that the variables $\mathrm{u}, \mathrm{v}$ are, by definition, fractions and cannot exceed unity; consequently the bounded nature of the solution should follow from the model itself. Notice too that it is the parameter $\eta$ which eliminates cycles as possible trajectories.

The Easter Island mystery: An interesting application of the Predator Prey Model is the paper by Brander and Taylor ${ }^{[9]}$. The problem they seek to analyze is the mystery of the island in the Pacific called Easter Island. Briefly (The interested reader is referred to Brander and Taylor $^{[9]}$ and references mentioned therein for details), the mystery is the following: when contact with European Civilization was established in the eighteenth century, it was also realized that the island must have been not only richer but also more populous in the past. This inference was based on the existence of huge statues carved from volcanic stones; some of the larger ones which could be moved weighed as much as 80 tons while the largest, lying unfinished in a quarry weighed as much as 270 tons. The puzzling aspect was that the Stone Age civilization found in the island in 1722 did not have the skills required for either producing these statues or moving them over any substantial distances. And the population seemed to be inadequate for moving the larger statues from the single quarry where they must have been mined: thus they lacked the brute force as well. The people of Easter Island, at that point of time, had no clue how the statues had been moved. The island has been extensively studied and analyzed and while there were no trees capable of making tools in 1722, subsequent geological studies established that the island must have been inhabited by Polynesians as early as 400 AD when there were large palm trees and extensive forest cover. These must have been cut down at the time of initial settlement to build boats and catch fish and this has been supported by the archaeological finding of fish bones. The population thrived, and may have found time for leisure activities which may have involved carving and moving statues.

Based on this hypothesis, a model is developed where the populace is treated as the predator and the resource base as the prey. Initially, the population was small and the resource base was plentiful and consequently, the population thrived; over time the resource base got depleted to the point that it could not 
regenerate itself and that led to the decline in the population. More specifically, by $900 \mathrm{AD}$ a substantial reduction in forests had taken place; the statues were carved between 1100 and 1500; by about 1400, the forest cover was almost completely depleted. Apparently with the rapid depletion of the resource base, the diet had also changed (less fish) with lower protein intake; soil erosion had taken place due to the same reason and this had contributed to a reduction in water retention which in turn had led to reducing productivity of land. Population peaked at around 1400 AD and then started to decline. The Europeans came in some three hundred years later and found the island impoverished and the population scarce.

These preliminary comments are insufficient to provide a complete picture but our purpose is much more limited: we wish to analyze the equations developed to explain this phenomenon. The following two equations are derived Eq. (11 and 12):

$$
\dot{S}=S\left\{r\left(1-\frac{S}{K}\right)-\alpha \beta L\right\}
$$

And:

$$
\dot{\mathrm{L}}=\mathrm{L}\{\mathrm{b}-\mathrm{d}+\phi \alpha \beta S\}
$$

In these equations, $S(t)$ stands for the resource stock at $\mathrm{t}$ and it grows at a natural logistic $\mathrm{r}$ rate minus the amount harvested $\mathrm{H}(\mathrm{t})$; assuming a Ricardian production set-up, this is seen to be $\alpha \beta \mathrm{L}(\mathrm{t}) \mathrm{S}(\mathrm{t})$ where $\mathrm{L}(\mathrm{t})$ stands for the population at time $\mathrm{t}$; the population growth rate is determined through the net birth rate b-d plus an allowance made for the fertility variation due to consumption per-capita of the resource $\phi \mathrm{H}(\mathrm{t}) / \mathrm{L}(\mathrm{t})=$ $\phi \alpha \beta S(t)$ : this is the Malthusian component.

The system made up of (11) and (12) is of course a Lotka Volterra system, with $\mathrm{L}$ as the predator and $\mathrm{S}$ as the prey; it is a special case of the system (3) with $\mathrm{M}=$ $\{\mathrm{r}(1-\mathrm{x} / \mathrm{K}) \alpha \beta \mathrm{y}\}, \mathrm{N}=\{\mathrm{b}-\mathrm{d}+\phi \alpha \beta \mathrm{x}\}$ where we have used $\mathrm{x}$ for the prey, here the resource stock (S) and $\mathrm{y}$ for the predator which is the population $\mathrm{L}$; given that assumptions P1 and P2, hold, (it is given that b-d $<0$ : that is, if there is no forest resource, the population will decline over time. Recall also that $\mathbf{P 3}$ was not really necessary and in this case, apart from the fact that $\mathrm{N}_{\mathrm{v}}=$ 0 all other restrictions in $\mathbf{P 3}$ do in fact hold.

In the circumstances: there can be no cyclical behavior; the only cyclical behavior which may be exhibited in this set-up is during the approach to an $E_{3}$ equilibrium, the spiral around the equilibrium with the fluctuations dying out over time. It turns out that our Fig. 2 shows precisely this kind of behavior. This however is not actual cyclic or periodic behavior. What then may we conclude from this very interesting exercise? We note as follows:
The assumptions relating to Ricardo or Malthus are not crucial for any of the conclusion drawn. Under very general conditions (P1 and P2 hold and solutions be bounded are sufficient for the purpose), it may be shown that our conclusions hold viz., that either there is convergence to a no-predator equilibrium $\mathrm{E}_{2}$ if there is no equilibrium with both species present or there is convergence to the equilibrium with both species present, $\mathrm{E}_{3}$, whenever it exists. In any case, there can be no periodic or cyclical behavior.

If the initial position is one where the predators are small in number and the prey population is small, the passage to either $E_{2}$ or $E_{3}$ type equilibrium, may involve first rapid growth in the population of the predators during which the prey population shrinks and then a fall in the population of predators. In fact as a reference to Fig. 3B will indicate, $E_{2}$ is a saddle point whenever $E_{3}$ exists; note also that the predators $\mathrm{L}$ are measured along the vertical axis while the prey $\mathrm{S}$ is measured along the horizontal axis. So if the initial point is close to the horizontal axes, with $\mathrm{S}^{\mathrm{O}}$ less than at $\mathrm{E}_{2}$ then the path will veer away from the horizontal axis, given the equilibrium and whose only line of approach is along the horizontal axes. In other words, there would be growth in the population of $\mathrm{L}$ and $\mathrm{S}$ too initially, as documented in the case of Easter Island (The problem, if any, with the Brander and Taylor ${ }^{[9]}$ contribution lies in their statement of proposition 4 (iii) and (iv); the statement seems to indicate that whenever the characteristic roots are imaginary (condition (14) holds), the trajectory will necessarily be as in their Fig. 2 (p. 126); that this is one among the many possible alternatives may be noted from their paragraph just preceding proposition 5 on p. 127). In fact, the solution will cross the $\mathrm{M}(\mathrm{x}, \mathrm{y})=0$ line and it is possible that along the solution, a level of population is reached which is greater than the maximum associated with $\mathrm{E}_{3}$, which is the 'overshooting' that Brander and Taylor $^{\left[{ }^{[]}\right.}$refer to. Notice that this is fully captured in the general formulation.

The special nature of the resource in Easter Island is responsible for the other element of the mystery i.e., why this pattern of development was recorded in Easter Island only and not in the other islands of the Pacific. Apparently the palm trees of Easter Island were ones with much slower rates of growth than elsewhere. Positive growth in the horizontal direction will become rather sluggish as a result. As a result, one may expect the $\mathrm{M}(\mathrm{x}, \mathrm{y})=0$ line to shift inwards and the equilibrium would involve lower value for both the variables: for instance, this is what happens for the functional forms assumed in Brander and Taylor ${ }^{[9]}$.

The authors seem to reject the simple explanation for the "mystery" of Easter Island: the population so degraded the resource base that it became unable to sustain the population. The population was, to begin 
with, very small, reached a height of between 10,000 to 15,000 about $1400 \mathrm{AD}$ and the period 1400-1500 AD saw falling food consumption accompanied with decreasing statue building activity. This fall in the population is seen as "overshooting" what was the sustainable population. This "overshooting" is however during a passage to some equilibrium. Whenever there is convergence, as in the present set-up, a trajectory becomes indistinguishable from the limit after a certain point of time (Hirsch and Smale ${ }^{[14]}$ p. 275, second paragraph after the diagram). Finally, as a reference to Fig. 3B would make clear, the line $M(x, y)=0$ is downward sloping in the $\mathrm{x}-\mathrm{y}$ plane with the prey $\mathrm{x}$ on the $\mathrm{x}$-axes. Consequently, the sustainable population of preys at $E_{3}$, is less than the sustainable population of preys when there is no predator at $\mathrm{E}_{2}$; now the initial point of the entire process is likely to be somewhere in between, with a low population of predators: consequently the line $\mathrm{M}(\mathrm{x}, \mathrm{y})=0$ is breached and the population of predators increase; this increase could take them beyond the level at $\mathrm{E}_{3}$ only to crash eventually since the preys are unable to keep up with its rate of depletion. As we have argued above, the simple explanation together with the special feature of the resource base in Easter Island, even within the context of a general predator-prey model, appears to be capable of explaining 'observed' facts.

An example of robust cyclical behavior: We provide a numerical example where the results of Claim 12 are satisfied. As will become apparent, we use our experience from the exercises of the last sections to construct such an example.

We consider a variation in our assumptions P2 which allows us to consider the rates of growth of the prey and predators as follows Eq. (13):

$$
\dot{\mathrm{x}}=\mathrm{x}\left\{\mathrm{r}\left(1-\frac{\mathrm{x}}{\mathrm{K}}\right)-\beta \frac{\mathrm{y}}{\mathrm{x}+\mathrm{h}}\right\} \text { and } \dot{\mathrm{y}}=\mathrm{y}\left\{\frac{\mathrm{bx}}{\mathrm{x}+\mathrm{h}}-\mathrm{d}\right\}
$$

where, $\mathrm{r}, \mathrm{K}, \beta, \mathrm{h}$ and $\mathrm{d}$ are all positive constants.

It may be noted that there is a similarity with the Brander-Taylor ${ }^{[9]}$ formulation: the growth of the prey in isolation follows a logistic rule. In the presence of the predator, the former has to be adjusted by the amount harvested or killed, which is assumed to be proportional to the population of the predators. The difference lies in this factor of proportionality.

In the Brander-Taylor ${ }^{[9]}$ formulation, this rate was also proportional to the population of the predators but the factor was increasing without bounds with the population of the prey: it may be recalled that this term was $\alpha \beta \mathrm{L} \mathrm{S}$; Where $\mathrm{L}$ represented the predator and $\mathrm{S}$ the prey populations, respectively. On the other hand, now, the corresponding term is $\beta \frac{x}{x+h} y$ with $x, y$ denoting populations of prey and predator respectively. Now the factor of proportionality does not increase without bounds with the population of the preys; there is a clear upper bound, since the factor $\mathrm{x} /(\mathrm{x}+\mathrm{h})$ approaches unity as the population of preys become infinite.

The population of the predators now takes into account the birth-rate, $b$ being the natural birth-rate with plentiful prey and $d$ is the death-rate among the predators; but the birth rate is affected when prey is not plentiful; in fact, the same type of argument was used in the Brander-Taylor ${ }^{[9]}$ formulation; however the effect is through a decline in the net birth-rate.

Rewriting the system (13) as: $\dot{\mathrm{x}}=\mathrm{xM}(\mathrm{x}, \mathrm{y}), \dot{\mathrm{y}}=\mathrm{yN}(\mathrm{x}, \mathrm{y})$ we note that now $\mathrm{M}(\mathrm{x}, \mathrm{y})=$ $\mathrm{r}(1-/ \mathrm{k})-\beta \mathrm{y} /(\mathrm{x}+\mathrm{h})$ and $\mathrm{N}(\mathrm{N}, \mathrm{y})=\mathrm{bx} /(\mathrm{x}+\mathrm{h})-\mathrm{d}$ so that $\mathrm{M}_{\mathrm{y}}$ $<0, N_{x}>0$; however $M_{x}$ could be either positive or negative. This is how $\mathbf{P 2}$ has been modified (For the system (3), $\mathbf{M}_{\mathrm{x}}<0$ and hence allowed the use of Dulac's criterion for ruling out the presence of cycles while admitting "social phenomenon"). The non-trivial equilibrium for this system is given by:

$$
x^{*}=\frac{d h}{b-d}, y^{*}=r\left(1-\frac{x^{*}}{K}\right)\left(x^{*}+h\right) / \beta
$$

Thus for a meaningful non-trivial equilibrium, we must have $\mathrm{b}>\mathrm{d}$ and in addition, we must have $\mathrm{K}(\mathrm{b}$ d)> $(b+d) h$ and we take it that this is so. At this equilibrium the Jacobian of the system is given by:

$$
\left(\begin{array}{cc}
x^{*} M_{x}\left(x^{*}, y^{*}\right) & x^{*} M_{y}\left(x^{*}, y^{*}\right) \\
y^{*} N_{x}\left(x^{*}, y^{*}\right) & 0
\end{array}\right)
$$

Notice that given the signs indicated earlier, the determinant is positive while the trace is of ambiguous sign. Notice too that the trace is given by:

$$
x^{*} M_{X}(.)=x^{*} \frac{K \beta y^{*}-\left(x^{*}+h\right)^{2}}{K\left(x^{*}+h\right)^{2}}
$$

After some simplification, it may be shown that the sign of the trace depends on the sign of $\mathrm{K}-\mathrm{h} 22_{\mathrm{x}}{ }^{*}$ and hence at the nontrivial equilibrium, either both of the characteristic roots have their real parts positive; or both have their real parts negative. Consider what happens when the trace happens to be positive: Notice that $\mathrm{x}^{*}$ is independent of the parameter $K$ so, keeping all the other parameters fixed, we need to choose a large enough value for $K$ for this to happen. Clearly then, the non-trivial equilibrium is a source.

Consider then, the case when $\mathrm{K}>\mathrm{h} 2{ }_{\mathrm{x}}{ }^{*}$; in other words, $\mathrm{K}(\mathrm{b}-\mathrm{d})>(\mathrm{b}+\mathrm{d}) \mathrm{h}$. The Poincaré-Bendixson Theorem, if applicable, would imply the existence of a closed orbit. 


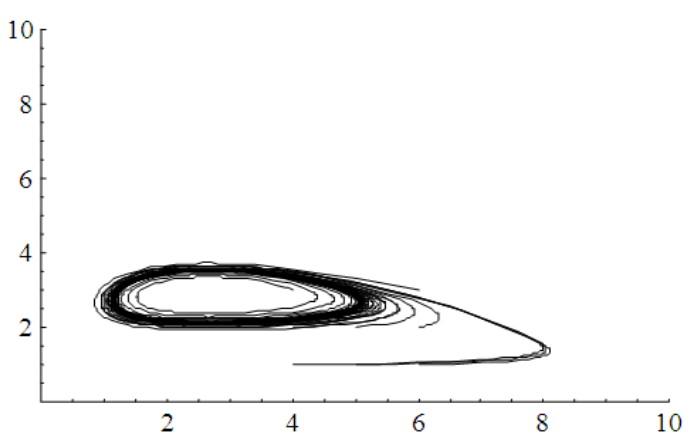

Fig. 4: Robust cyclic behavior

We need to check whether any equilibrium can be approached and then check whether trajectories are bounded for this purpose. Notice that the only other equilibria for the system (13) are given by $(0,0)$ and $(\mathrm{K}, 0)$; computation of characteristic roots of the Jacobian of the system at these equilibria implies that $(0,0)$ is a saddle-point and if $b+d>1$, then so is $(K, 0)$. The following may be checked:

If the initial point $\left(\mathrm{x}^{\mathrm{O}}, \mathrm{y}_{\mathrm{O}}\right)>(0,0)$ none of these equilibria can be approached. Trajectories remain bounded.

Consequently the only possibility is a limit cycle around the non-trivial equilibrium. Notice also that small perturbation of the system is unable to dislodge the cyclical behavior of trajectories. Thus to clinch matters we need to establish the validity of the items noted above.

For the first, it is best to consult the following figure, where we have considered the phase plane of the system (13), for appropriate values of the various parameters: $r=$ $10, \beta=20, b=4, d=2, h=4$ with appropriate units (All but the last are measured in number per year. The last is a stock and is measured in numbers, say millions). Notice that $\mathrm{K}$ has been left unspecified.

The case we are interested in, consists of requiring $\mathrm{K}>12$ (this would make the interior equilibrium unstable) $(\mathrm{K}=$ 12 is a point of bifurcation at which point, the non-trivial equilibrium loses stability and an attracting closed orbit emerges); the situation for $\mathrm{K}=16$ is captured below.

While the above is a computer generated figure and cannot be taken for an analytical proof, we note that the axes $\mathrm{x}=0, \mathrm{y}=0$ are trajectories and cannot be crossed; and the only trajectories which approach the saddlepoint equilibria are these trajectories. The non-trivial equilibrium being a source cannot be approached. Hence no trajectory, with a strictly positive initial point, can approach equilibrium, as claimed.

To show that any trajectory beginning from an initial positive configuration remains bounded, notice the following: denoting the solution to (13) from an arbitrary $\mathrm{z}^{\circ}=\left(\mathrm{x}^{\circ}, \mathrm{y}^{\circ}\right)>(0,0)$ by $\varphi_{\mathrm{t}}\left(\mathrm{z}^{\circ}\right)=\left(\varphi_{\mathrm{tx}}\left(\mathrm{z}^{\circ}\right), \varphi_{\mathrm{ty}}\right.$ $\left.\left(\mathrm{z}^{\circ}\right)\right)$; note that $\varphi_{\mathrm{tx}}\left(\mathrm{z}^{\circ}\right)<\mathrm{K} \forall \mathrm{t}>0$ since $\varphi_{\mathrm{tx}}\left(\mathrm{z}^{\circ}\right)=\mathrm{K} \Rightarrow \mathrm{x}=$ $\varphi_{\mathrm{tx}}\left(\mathrm{z}^{\circ}\right)<0$; thus unbounded behavior, if possible, may arise only if $\varphi_{\text {ty }}\left(\mathrm{z}^{\circ}\right) \rightarrow \infty$; if this were to be the case, $\dot{\mathrm{x}} \rightarrow-\infty$ and for all $\mathrm{t}>\mathrm{T}$, say, $\dot{\mathrm{x}}<-\delta$ for some $\delta>0$. This contradicts the fact that $\varphi_{\mathrm{tx}}\left(\mathrm{z}^{\circ}\right)>0 \forall \mathrm{t}$. Thus the solution $\varphi_{\mathrm{t}}\left(\mathrm{z}^{\circ}\right)$ remains within a bounded region of the positive quadrant. Thus the $\omega$-limit set is non-empty and does not contain any equilibrium when $\mathrm{K}(\mathrm{b}-\mathrm{d})>$ $(b+d) h$; an appeal to Poincaré-Bendixson Theorem establishes that there must be a limit cycle.

That this is an example of robust cyclical behavior may be gauged from the fact that small perturbation in parameter values would maintain the local properties of the three equilibria (Notice too that the bounded nature of trajectories follow regardless of parameter values.) and also maintain the crucial inequality $K(b-d)>(b+d)$ $\mathrm{h}$. Hence the limiting periodic behavior would also be maintained. In fact, the diagram constructed shows that even with initial points being different, the solution settles down into a closed orbit (the limit cycle).

\section{CONCLUSION}

The Predator-Prey Models thus provide a framework, where a wide variety of results are possible: not only one may get convergence as in the set-up described by assumptions P1-P3, but also one may obtain periodic behavior if the equations of motion are described by (1) or by equations such as the ones in the last section. The current paper shows what is needed to get a proper periodic or persistent cyclic behavior. First of all a Predator-Prey model with two species by definition would require that $\mathrm{M}_{\mathrm{y}}<0$ and $\mathrm{N}_{\mathrm{x}}>0$ where $\mathrm{x}, \mathrm{y}$ are the population of the preys and predators respectively and $\mathrm{M}($.), N(.) respectively define their rates of growth. The differences in conclusions arise from what we assume about $\mathrm{M}_{\mathrm{x}}, \mathrm{N}_{\mathrm{y}}$ i.e., how the rates of population growth are affected by their own population levels, other things being held constant. In fact, we have shown how crucial the role of the so-called 'social phenomenon' is for the dynamic conclusions. To distinguish our conclusions from the conclusions in Kolmogorov ${ }^{[12]}$ or as reported in Freedman ${ }^{[13]}$, our method allows us to conclude that it is this aspect which is crucial. In addition, it should be pointed out that the fact global stability results for special forms of predator-prey models have been appearing even recently ${ }^{[11]}$, suggest that there are still matters to be investigated.

To remind readers: the simple model (1) ensures that $M_{x}=N_{y}=0$; it may be shown that this is enough to generate the closed orbit in the original Predator-Prey Model i.e., the linearity assumed in the rates of growth in (1) are really not required. If we allow these partial derivatives viz., $\mathrm{M}_{\mathrm{x}}, \mathrm{N}_{\mathrm{y}}$ to be negative (This forces "social phenomenon" to be always negative. As we have discussed above, to rule out periodic behavior, it is enough if these partial derivatives have the same weak signs and be not identically zero.), we are in the situation described by assumptions P1 and P2 and the earlier closed orbits disappear. This result is obtained under general conditions. 
If one allows for changes in the signs of these particular partial derivatives to vary in sign over the domain under consideration only then, may one hope to obtain a description of persistent cyclic behavior. It should be noted that this is not sufficient, since even with variation in the sign of these partial derivatives, one may get convergence under some conditions, as noted by Hsu and Huang ${ }^{[11]}$. They study the following system:

$$
\dot{\mathrm{x}}=\mathrm{x}\left\{\mathrm{g}(\mathrm{x})-\mathrm{p}(\mathrm{x}) \frac{\mathrm{y}}{\mathrm{x}}\right\} \text { and } \dot{\mathrm{y}}=\mathrm{y}\left\{\delta-\beta \frac{\mathrm{y}}{\mathrm{x}}\right\}
$$

The functions $\mathrm{g}(\mathrm{x}), \mathrm{p}(\mathrm{x})$ satisfy:

$\mathrm{g}(1)=0, \mathrm{~g}^{\prime}(\mathrm{x})<0 \forall \mathrm{x}>0, \mathrm{p}(0)=0, \mathrm{p}^{\prime}(\mathrm{x})>0 \forall \mathrm{x}>0$

The above assumptions serve to keep the solution in a bounded region: $0<x<1,0<y<\delta / \beta$; the claim is that if, in addition, one has:

$\left(\mathrm{x}-\mathrm{x}^{*}\right)\left\{\frac{\mathrm{xg}(\mathrm{x})}{\mathrm{p}(\mathrm{x})}-\mathrm{y}^{*}\right\}<0,0<\mathrm{x}<1, \mathrm{x} \neq \mathrm{x}^{*}$

Then the equilibrium $\left(\mathrm{x}^{*}, \mathrm{y}^{*}\right)$ is globally stable. Notice that in this case, while our crucial P1 holds, P2 does not, and if the additional requirement stated above is met, then global stability of the interior equilibrium is claimed.

Our example provides a description of a situation where we allow social phenomenon but do not restrict that social interaction to be always negative; this establishes a robust cyclical behavior.

Important implications of our results are exhibited by the considered applications: in the case of the Goodwin type models analyzed by Flaschel ${ }^{[15]}$ : It is the money illusion term which destroys periodic behavior; none of the other assumptions made in that paper is really required; similarly for the Brander-Taylor ${ }^{[9]}$ contribution, the specificities imposed through the Ricardo-Malthus assumptions are not necessary as well. The simple setting for the Easter Island, with the resource base as prey and the population as predator, and 'social phenomenon' exhibited in the growth rate of the prey, together with the fact that the resource base in Easter Island had special characteristics, provide an explanation for the Easter Island Mystery.

\section{ACKNOWLEDGEMENT}

Preliminary version of results reported here were circulated in the Discussion Paper titled "Competitive equilibria: Convergence, cycles or chaos" completed during the author's visit to the Institute of Social and Economic Research, Osaka University, August 2002July 2003; support provided is gratefully acknowledged. The results were reported in an invited lecture at the $4^{\text {th }}$ MME Conference of the Indian Statistical Institute,
Kolkata, December 2004. The author is grateful to the organizers, Uday Bhanu Sinha and Manipushpak Mitra for their invitation. The author is indebted to Partha Gangopadhyay for his help and support.

\section{REFERENCES}

1. Kydland, F. and E. Prescott, 1982. Time to build and aggregate fluctuations. Econometrica, 50: 1345-1371.

2. Schumpeter, J.A., 1939. Business Cycles: A Theoretical, Historical and Statistical Analysis of the Capitalist Process. McGraw Hill, New York.

3. Wicksell, K., 1936. Interest and Prices: A Study of the Causes Regulating the Value of Money. Macmillan Co., London.

4. Goodwin, R.M., 1967. A Growth Cycle. In: Socialism, Capitalism and Economic Growth, Essays Presented to Maurice Dobb, Feinstein, C.H. (Ed.), Cambridge University Press, London.

5. Goodwin, R.M., 1982. Essays in Economic Dynamics. MacMillan and Co., New York.

6. Samuelson, P.A., 1972. Generalized predator-prey oscillations in ecological and economical equilibrium. Collected Scientific Papers Paul A. Samuelson, 3: 487-490.

7. Samuelson, P.A., 1972. A Universal Cycle? In The Collected Scientific Papers of Paul A. Samuelson, 3: 473-486.

8. Mukherji, A., 2005. Robustness of Closed Orbits in a Class of Dynamic Economic Models, in Economic Theory in a Changing World, Policy Modeling for Growth edited by Sajal Lahiri and Pradeep Maiti, Oxford University Press, Delhi.

9. Brander, J.A. and M.S. Taylor, 1998. The simple economics of Easter Island: A Ricardo Malthus model of renewable resource use. Am. Econom. Rev., 88: 119-138.

10. Cressman, R., W. Morrison and J.F. Wen, 1998. Evolutionary dynamics of crime. Canadian $\mathrm{J}$. Econom., 31: 1101-1117.

11. Hsu, S.B. and T.W. Huang, 1995. Global Stability for a class of Predator-Prey Systems. SIAM J. App. Mathematics, 55: 763-783.

12. Kolmogorov, N., 1936. Sulla teoria di Volterra della lotta per l'esistenza, Giornelle dell'Istituto Italiano degli Attuari, 7: 74-80.

13. Freedman, H.I., 1980. Deterministic Mathematical Models in Population Ecology. Marcel Dekker Inc. New York.

14. Hirsch, M.W. and S. Smale, 1974. Differential Equations, Dynamical Systems and Linear Algebra. Academic Press: New York.

15. Flaschel, P., 1984. Some stability properties of Goodwin's growth cycle, a critical elaboration. Zeitschrift für Nationalökonomie. J. Econom., 44: 63-69. 